\title{
Care needs and preferences from the perspective of COVID-19 patients: A qualitative study
}

\author{
Malihe Sadat Moayed ${ }^{1}$, Esmail Heidaranlu ${ }^{1}$, Akram Parandeh $^{2 *}$ (1)
}

Received: 11 Oct 2020

Published: 19 May 2021

\section{Abstract}

Background: The COVID-19 epidemic is one of the major health problems worldwide due to its inconceivable spreading power and potential damage. Given the increasing prevalence of the disease, the identification of care needs and preferences of patients could play an important role in providing effective training and caring programs. This study was conducted to explain the preferences and needs of care based on the experiences of patients with COVID-19.

Methods: This qualitative study with a content analysis approach was performed in 2 months at a referral general hospital and quarantine centers of COVID-19 in Tehran, the capital of Iran, in 2020. The participants consisted of 15 COVID-19 patients selected through purposive sampling. The data analysis was performed using the conventional content analysis method according to the procedure proposed by Graneheim and Lundman.

Results: The results were classified into 5 main categories: (1) access to desirable care and comfort services; (2) access to education and information from credible sources; (3) access to specialized care; (4) support social needs; and (5) need for deep emotional interactions.

Conclusion: According to our results, identifying priorities and care needs from the perspective of patients with COVID-19 can help improve knowledge, reduce unrealistic patient concerns, and improve emotional interactions between patients and health care providers.

Keywords: Coronavirus Disease 2019, Health Preferences, Iranian Health System, Needs Assessment, Qualitative Study

Conflicts of Interest: None declared

Funding: None

*This work has been published under CC BY-NC-SA 1.0 license.

Copyright $($ Iran University of Medical Sciences

Cite this article as: Moayed MS, Heidaranlu E, Parandeh A. Care needs and preferences from the perspective of COVID-19 patients: A qualitative study. Med J Islam Repub Iran. 2021 (19 May);35:64. https://doi.org/10.47176/mjiri.35.64

\section{Introduction}

The COVID-19 epidemic, caused by a novel beta coronavirus called SARS-CoV-2, emerged in China in late December 2019 and spread all over the world in less than 4 months. On March 11, 2020, the World Health Organi-

Corresponding author: Dr Akram Parandeh, akramparandeh2000@bmsu.ac.ir

1. Trauma Research Center, Faculty of Nursing, Baqiyatallah University of Medical Sciences, Tehran, Iran

2. Medicine, Quran and Hadith Research Center, Faculty of Nursing, Baqiyatallah University of Medical Sciences, Tehran, Iran zation (WHO) declared COVID-19 as a pandemic, stressing the danger of this outbreak for public health (1).

Outbreaks of i nfectious diseases have always been difficult health challenges, threatening the wellbeing of peo-

$\uparrow$ What is "already known” in this topic:

Paying attention to patients' needs is one of the most important factors affecting their recovery.

$\rightarrow$ What this article adds:

Patients with COVID-19 have extensive needs in the treatment environment. Among these, attention to emotional needs plays an important role from the perspective of patients.

$\square$ Poor clinical conditions require the presence of competent and specialized treatment staff to provide specialized care compared to other patients.

$\square$ Due to the extensive information from various and erroneous sources, it is necessary to provide the staff with education from credible sources. 
ple not only directly, but also indirectly through their effects. In the early stages of the spread of SARS and MERS, there were many reports of a wide range of outbreak-induced psychological problems, including depression, anxiety, panic attacks, psychotic excitement, psychotic disorders, and even suicide $(2,3)$. For these outbreaks, the lack of participation of patients in care decisions, poor isolation during hospitalization, and failure to respond to patients' requests have been identified as the most important management challenges in the medical sector (4). The top preferences from the perspective of hospitalized patients, however, are believed to be nursepatient communication and interactions, access to personal protective equipment, and comfort during hospitalization $(5,6)$.

Today, the quality of care in health care systems has drawn even further attention and scrutiny (7). Naturally, the quality of hospital processes and services cannot be improved without the active pursuit of patient satisfaction and particular attention to patients' needs and expectations in terms of comfort as well as clinical services (8). Thus, health systems should be committed to identify and meet the psychological, physical, and other needs of patients during the treatment process (9-11). Patient care needs are a complex, multifaceted, and contextual concept that are discussed in the literature (12).

Psychological, therapeutic, and care needs of the patients have received the most attention in the literature (13). The surprising emergence and spread of the COVID19 pandemic, the unknown and ambiguous behavior of the virus, the poor knowledge of health care personnel of all levels about treatment, care methods, and requirements for these patients have created a sense of profound uncertainty and anxiety in several health care personnel and patients, especially in the early days (14).

According to the WHO, the effects of COVID-19 will certainly persist until the end of 2020, and its outbreak might very well return to plague the world in the coming years (15). Therefore, identifying the basic needs of COVID-19 patients, determining their satisfaction, and giving peace of mind during the treatment can have a deep impact on the quality of treatment and care services.

Considering the shortage of in-depth studies on the educational, health, and care needs and requirements of COVID-19 patients and the fact that quantitative research methods fail to provide deep insights into beliefs, thoughts, and attitudes of their subjects, the use of qualitative research methods to identify the needs and preferences of COVID-19 patients may be able to greatly contribute to progress in this field. Indeed, qualitative studies discover the needs of patients from their own words, thereby providing deeper and more objective insights into the unique experiences of these people (16). In view of the above, it seems necessary to increase our understanding of the experiences of patients with coronavirus and consequently the clinical application of research findings in nursing and psychological interventions. The present study was part of a large study on Iranian COVID-19 patients, which, in this part, aimed to identify the care needs of these patients from their perspective and preferences while receiving treatment.

\section{Methods}

This qualitative study with a content analysis approach was conducted in 2020 to determine the care and treatment needs of Iranian COVID-19 patients. The study was performed in 2 months in a referral general hospital and quarantine centers of COVID-19 patents in Tehran, the capital of Iran, in 2020. The sample consisted of 15 COVID-19 patients selected through purposive sampling. The inclusion criteria were as follows: having a positive test of COVID-19, being without any physical or mental disability, having the ability to communicate and the willingness to cooperate. For triangulation in data sources, some interviews were performed with the health care workers. The participants were selected for maximum possible diversity in terms of age, sex, education, and history of admission in medical and recovery units. The COREQ checklist was used to report the study. The data were collected through semi-structured one-on-one interviews with 6 patients, 2 focus group interviews with a homogeneous group of 4 to 6 people, and telephone interviews with COVID-19 patients being treated in medical and recovery units. The time and place of interviews were determined by the mutual agreement of the patients and the researcher. Each interview lasted 15 to 30 minutes. Participants were COVID-19 patients who were able to fully articulate their experiences. The interviews in medical and recovery units were conducted in a convenient location in terms of noise, light, and privacy (lounge or private rooms). The group interviews were conducted with the attendance of participants who were seated in a circle, 1 researcher in charge of managing the meeting, and 1 researcher taking notes of the issues raised during the discussion.

At the beginning of each interview, the researcher introduced herself and explained the objectives of the study. The informed written consent of all the participants was obtained to record the interviews.

During the interviews, verbal communication and nonverbal behaviors, eye contact, and changes in facial expressions were noted and recorded. Interview sessions were held in the mornings or evenings and scheduled at the convenience of the participants. To keep the interviews uniform, some guide questions were prepared for all the sessions. Each interview started with the general question "Would you please tell us about your experience with COVID-19?" and continued with further specific questions, such as "What were/are your health care needs during hospitalization?" "What problems did/do you face?" etc. When necessary, the researcher asked exploratory questions, such as "Please explain more" or "What do you mean?"

The interviews continued until data saturation. Data analysis was performed using the conventional content analysis method according to the procedure proposed by Graneheim and Lundman. Primarily, the interviews were transcribed verbatim and reviewed several times. Subsequently, words, phrases, or paragraphs that could be meaning units were identified and coded. The codes were 
compared to identify the similarities and differences and were then classified into more abstract categories. The members of the research group continuously discussed the choice of codes and their categorization into themes and subthemes until reaching a consensus in this regard. Data collection continued until saturation in each category, after which the results were typed and coded manually.

Lincoln and Guba's criteria were used to improve the accuracy and rigor of the data. To ensure its credibility, the researchers spent enough time on data collection, created a sample with maximum possible diversity (age, education, and sex), remained engaged with the data for a prolonged period of time, and discussed the data and interpretations with the participants (member checking). To ensure dependability and reliability, the methods were shared with colleagues for review. Concerning the confirmability of the findings, the researchers tried to not allow their biases to impact data collection and analysis. Finally, to ensure transferability, the researchers tried to provide a detailed description of the processes of data collection, encoding, and analysis.

\section{Results}

Our participants herein were mostly men $(87 \%)$ with a mean age of $50.85 \pm 6.02$ years. Approximately half of the participants $(54 \%)$ had higher education. The mean duration of infection with COVID-19 was $7.57 \pm 5.15$ days.

After identifying and reviewing the codes, they were categorized, based on their similarities into 5 main categories and 15 subcategories. The main categories obtained from this process are (1) access to desirable care and com- fort services; (2) access to education and information from credible sources; (3) access to specialized care; (4) support social needs; and (5) need for deep emotional interactions. The descriptions of these categories and their subcategories are presented as follows: (Diagram 1).

\section{Access to Desirable Care and Comfort Services}

This refers to the need of COVID-19 patients to receive quantitatively and qualitatively acceptable care from committed and skilled health care workers to have sufficient access to protective equipment, to be provided with a safe and healthy physical space, and to be cared for in terms of basic needs. These subcategories are further explained in the following.

\section{A) Need for Skilled and Committed Health care Work-} ers

The presence of a skilled and committed medical team is a key determinant of the efficacy of treatments and patient recovery. Access to specialized care provided in a committed manner, particularly for diseases that do not have any definitive or approved treatment, can play a significant role in the prognosis of patients.

In this regard, Participant No. 1 (a 61-year-old male) said:

"I cough for hours, but no one cares! They just give me oxygen. Shouldn't a doctor come check me? When I tell the nurses, they say we'll call the doctor, but your condition is normal."

Having a sufficient number of health care workers to meet the treatment and care needs of patients is extremely

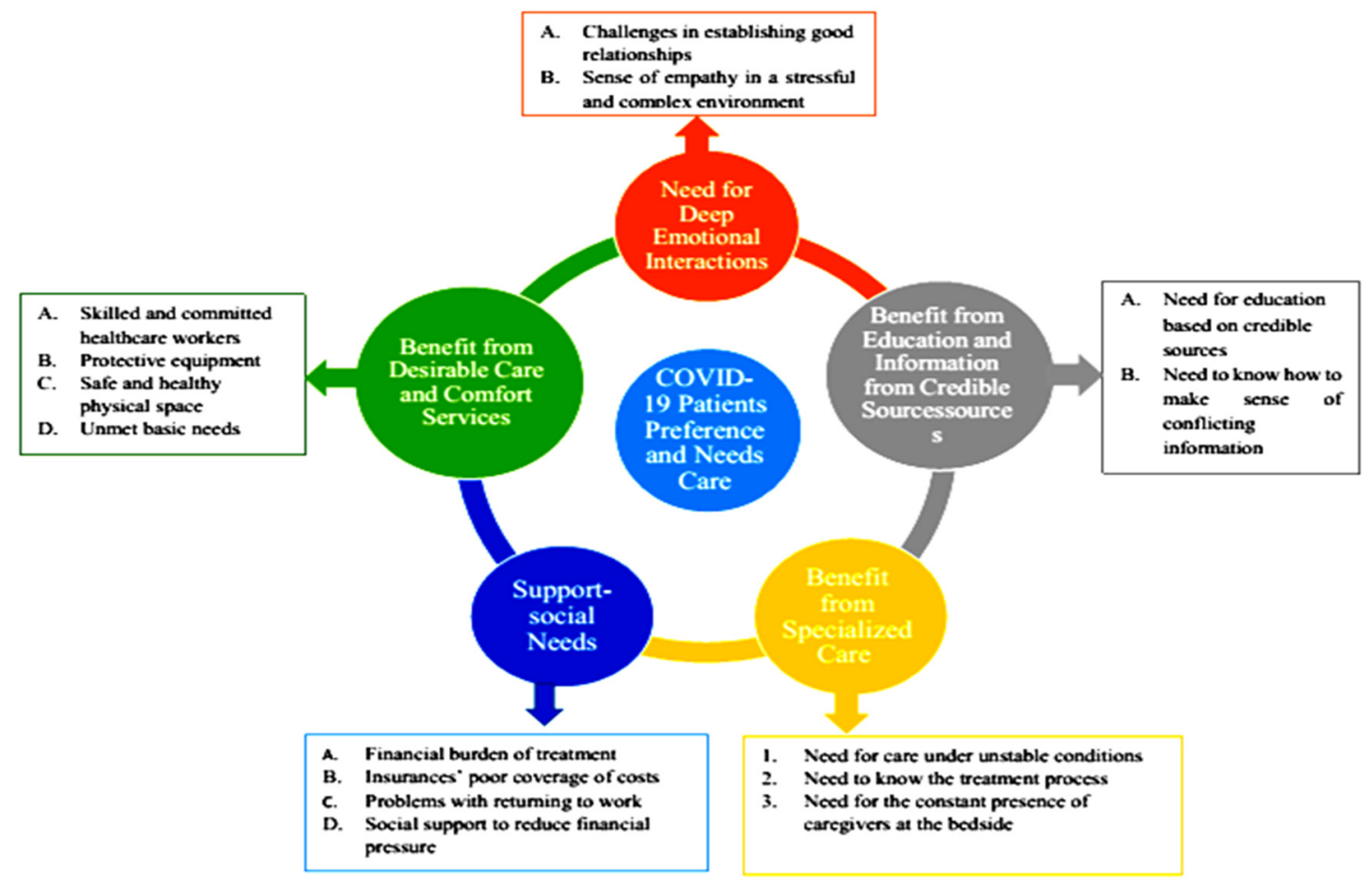

Diagram 1. Five categories of needs and preferences from perspective of COVID-19 patients 
important for ensuring that all COVID-19 patients receive the necessary care for this disease.

In this regard, Participant No. 5 (a 49-year-old male) stated:

"Our problem is that there is not enough manpower in the ward. In the last shift, the mother of one of the nurses died in this very same ward, so she left. My nurse, who already had 3 patients, now had to serve 4 patients; 2 of them were critically ill and were on respirator. The way it is, often, they do not have enough time to look after all of us."

\section{B) Protective Equipment}

Since the commitment to cautionary protocols, such as using masks, frequent hand washing, and other protective measures are essential for protecting healthy individuals and limiting the transmission, access to modern and highquality protection equipment for COVID-19 patients as well as health care workers is one of the most important requirements.

Regarding the need for appropriate equipment to control the disease and receive proper care, Participant No. 4 (a 53-year-old female) said:

"When I ask for a mask, they don't give me one; they say you must put on your oxygen mask. When I say, 'what I keep in front of my mouth when I should go to the bathroom, ' they say we are short of masks...."

Additionally, Participant No. 2 (a 52-year-old male) stated:

"There are many shortages. I cough all the time. They say that you should put on these oxygen masks 24 hours a day. When I say give me a mask to prevent spreading the virus. ... Nurses and staff say we don't even have enough masks for ourselves!"

\section{C) Safe and Healthy Physical Space}

Creating a well-ventilated environment with good air circulation is one of the ways to control the transmission of the disease. Access to such a safe and healthy space was another request of patients.

In this regard, Participant No. 5 (a 49-year-old male) said:

"The ward does not have proper ventilation. You can leave the terrace door open to allow the airflow through, like a corridor, but they don't do this..."

"A few days ago, some guys from the Health Ministry sprayed the whole area, the beds, the floors, the tables, everything; but they did not return..., I think this should be repeated every day."

In addition, a participant in Focus Group 1 (a 48-yearold male) stated:

"The recovery area is not suitable for hospitalization. We don't have adequate lighting. The space is confined, and everything is squeezed. It is not well ventilated at all. It is very cold at night and there isn't enough oxygen during the day."

Participant No. 4 in Focus Group 2 (a 51-year-old male) also said:

"I have a lumbar disc. It's really hard for me to rest on these beds. It is a hard bed with a thin blanket. Also, the beds that don't have side rails, so we may fall dawn at night...".

\section{D) Unmet Basic Needs}

COVID-19 changes the sense of taste and smell and significantly affects the appetite of the patients. Therefore, attention to basic needs is a preference for these patients. One of the common complaints made in the interviews was the lack of enough specialized attention to basic needs such as diet.

For example, Participant No. 3 (a 37-year-old male) stated:

"Food quality is terrible; the foods they provide have nothing to do with the disease. The disease also makes it hard to eat here."

As patients overcome the acute condition of the disease and start to recover, their nutritional needs also increase, which is why it is recommended to provide more meals during the recovery period.

In this regard, Participant No. 1 in Focus Group 2 (a 32year-old male) said:

"The hours of the meals are not good. For example, we have to stay hungry from 7 PM to 7 AM. Instead of eating my entire dinner at once, I eat it slowly until 11. But we don't have anything to heat food. So, if you want to wait a few hours, you will be eating cold food."

In view of the long stay of patients and their isolated conditions, it is important to pay attention to their basic hygienic needs as well.

For example, Participant No. 4 (a 53-year-old female) stated:

"Bathrooms with proper appliances are very important for hygiene. Unfortunately, the space provided is not suitable for women."

\section{Access to Education and Information from}

\section{Credible Sources}

This category refers to the need for patients' education based on credible information about the disease and how it can be prevented, controlled, or treated and the need to help patients make sense of the conflicting information that they may receive from different sources.

\section{A) Need for Education Based on Credible Sources}

Providing COVID-19 patients with up-to-date credible information, particularly through physicians and nurses, and keeping them involved during hospitalization can prevent possible complications and facilitate recovery by enhancing self-care.

Regarding patients' need for education, Participant No. 5 (a 49-year-old) stated:

"I wish there was a law forcing doctors or nurses to give a scientific explanation of our situation to our families so that they would not be subjected to such stress."

\section{B) Need to Know How to Make Sense of Conflicting In-} formation

Today, social media and networks play a pivotal role in disseminating both reliable and unreliable information. Naturally, the dissemination of conflicting information 
through these networks and other web services can confuse patients.

For example, Participant No. 5 (a 49-year-old) said:

"Every day, false news from websites makes patients and their families even more confused. Every day, they introduce a new way to control or treat the virus, which later you find out is completely baseless. You really don't know which is true and which is not."

Participant No. 3 (a 37-year-old male) stated:

"The way it is, we're really confused, we don't know which news is right and which is false, we can't trust social media."

\section{Access to Specialized Care}

One of the most important needs of patients with COVID-19 was to receive specialized care that includes comprehensive care under unstable conditions, knowledge of the treatment process, and the constant presence of caregivers at the bedside. These 2 subcategories are described below.

\section{A) Need for Care Under Unstable Conditions}

One of the confirmed features of COVID-19 is clinical instability and sudden changes in hemodynamic conditions, which makes the patient dependent on extensive care.

In this regard, Participant No. 2 (a 52-year-old male) said:

"... Our situation is so bad that we don't notice the passing of time. Nurses constantly obtain blood pressure and temperature. Doctors constantly examine us. Sometimes I get physiotherapy. Our condition is constantly changing; the patients in my left and right side of the bed were good in the morning, but both died on the same day....".

\section{B) Need to Know the Treatment Process}

One of the demands of patients and their families was to keep being updated about their conditions. Since families are not allowed to visit their patients in medical wards, they are constantly anxious about the latest condition of their patients.

In this regard, a patient in Focus Group 1 (a 21-year-old male) stated:

"My family members called the ward for several days in a row. The nurses kept saying to them 'your patient is like yesterday; he hasn't changed at the moment.' It's important for the family to know about the patient's medical condition."

Participant No. 5 (a 49-year-old male) said:

"I'm not complaining about how these employees keep working in these difficult conditions, but I am not satisfied with the way doctors and nurses treat me. I don't know anything about the course of my illness. The doctor who examines me should at least try to explain my condition."

\section{C) Need for the Constant Presence of Caregivers at the} Bedside

The changing and critical conditions of certain patients require close and continuous care and reexamination to assess how the disease is progressing or regressing.

Participant No. 4 (a 53-year-old female) said:

“... I don't really understand the doctor's visit because I don't usually see a doctor come near my bed. I haven't seen the doctor in these few days; they just write something on my file from afar and leave..."

A patient in Focus Group 1 (a 32-year-old) stated:

"... In the morning, someone writes the name of a doctor and a nurse on the board above our head. Sometimes, I ask something from an employee, even a nurse, they just say wait for your nurse to come.... My medicine has run out and an alarm keeps going off in the machine.... they say the new medicine will start once your own nurse is here....".

\section{Support Social Needs}

The subcategories of support social needs of COVID-19 patients were as follows: the financial burden of treatment, insurance poor coverage of costs, problems with returning to work, and social assistance to reduce financial burden.

\section{A) Financial Burden of Treatment}

Since COVID-19 is an emerging and unknown disease and still does not have a definitive cure, patients must receive multiple treatments simultaneously, which substantially increases the financial burden of this condition.

Participant No. 6 (a 35-year-old nurse) said:

"There was no treatment protocol. Therapies were mostly experimental. Every day, a new treatment was recommended. One day, they recommend something which is rejected the next day and replaced with something else. These things cost a lot."

\section{B) Insurances' Poor Coverage of Costs}

The variety of treatments and drugs used on COVID-19 patients greatly increases the cost of treatments and services provided. Meanwhile, a number of drugs or treatment protocols are not covered by health insurance, imposing a great financial burden on patients.

Regarding the financial problems caused by poor insurance coverage, a patient in Focus Group 2 (a 25-year-old female) said:

"We have a problem with hospital bills. Should I bear the pain of the disease or the pain of hospitalization cost? They gave my family a prescription, saying that the hospital doesn't have the drugs, so you should get them from elsewhere. The prescription costs a lot and we have to pay out of pocket. Well, I have health insurance, but why isn't it covered by insurance? In this corona condition (referring to pandemic), should they send my family to fetch medicine?"

Additionally, Participant No. 2 in Focus Group 1 (a 61year-old male) stated:

"Fearing that their insurance will not cover them, many patients prefer to go home and risk getting their family infected, because they think it will cost a lot and they will not be able to handle it."

C) Problems With Returning to Work

Returning to work after recovery was one of the con- 
cerns of patients, especially those with nongovernmental jobs.

Participant No. 3 (a 37-year-old male) said:

"My main problem is my job; I am a worker; what should my family do financially? This brings me more anxiety than the disease itself. I am afraid of being fired by my employer."

\section{D) Social Support to Reduce Financial Pressure}

COVID-19 has negatively affected Iran in several ways, but it has also generated a sensation that all Iranians have a common destiny and that their wellbeing depends on the wellbeing of others.

In this regard, Participant No. 6 (a 35-year-old nurse) stated:

"Many patients have financial problems, but there is a sense of empathy that has caused people to help patients by bringing food or even paying medical expenses."

\section{Need for Deep Emotional Interactions}

One of the main elements of hospital care is to provide emotional support and peace of mind by establishing a good relationship with patients. One of the needs of COVID-19 patients was related to the lack of deep emotional interactions, which manifested in 2 subcategories: challenges in establishing good relationships and a sense of empathy in a stressful and complex environment.

\section{A) Challenges in Establishing Good Relationships}

One of the important determinants of the quality of care and consequently the outcome of treatment is whether there is a good relationship between health care workers and patients. This was also found to be an important need of COVID-19 patients.

In this regard, Participant No. 5 (a 49-year-old male) said:

"Nurses keep their distance and don't want to make any contact; although they wear space suites, masks, glasses, shield, gun, shoe cover, even have multilayered gloves, and the patient is also masked. This is running from the patient; this means taking an emotional distance!"

B) Sense of Empathy in a Stressful and Complex Environment

Empathy is an active effort to understand others and an emotional response to their situation. It is the ability to see things from another person's point of view and to behave in a way that heals the suffering of others in acts that often involves courage and philanthropy based on socially acceptable behaviors. Empathy can also be viewed as the opposite of being indifferent toward others. It could accelerate the improvement of patients' physical and mental conditions.

In this regard, a participant in Focus Group 2 (a 28year-old female) said:

"...Working for and serving coronavirus patients, who carry millions of viruses, requires love. This should not become pure duty. The person must remain in love with the work..."

Additionally, a participant in Focus Group 1 (a 36-year- old male) stated:

“...I enjoy how seminary students help out without any ego; it is very good. They just do their service lovingly. They try hard to make you comfortable, with pleasure. This is great in this situation, but they just come here for a few hours. I wish they were here all the time....".

\section{Discussion}

During the course of their disease and treatment, COVID-19 patients experience an acute and critical condition under which they develop certain needs that must be met with help from others, specifically health care workers. This study aimed to identify the care needs of these patients from their own perspective. After analyzing the collected data, this study identified 5 main categories of needs: (1) access to desirable care and comfort services; (2) access to education and information from credible sources; (3) access to specialized care; (4) support social needs; and (5) need for deep emotional interactions. In other words, the participants believed that the fulfillment of these needs should improve the treatment and care conditions of COVID-19 patients.

Meeting the needs and expectations of patients is one of the most important tasks of health care organizations, as it encourages patients to carry out physician orders correctly and in a timely manner, which works toward the main goal of the whole process, which is accelerating the treatment and recovery processes (17). Health care services are among a few groups of services that everybody needs yet may not necessarily desire (18). Nevertheless, patient satisfaction is an element of great importance in care and treatment service evaluations. Indeed, meeting the needs of patients in a way that results in their satisfaction determines whether the hospital is successful in properly delivering its services (19). In Iran's Health Sector Transformation Plan, hospital hoteling services have been defined in 7 main areas: quality of buildings and facilities, quality of equipment, quality of comfort, quality of cleaning and sanitation, quality of nutrition, quality of administrative affairs and workflow, and quality of human resources (20). In a study that examined the patients' views on how well their needs are met, it was reported that the quality of comfort, human resources, cleaning and sanitation services, hospital buildings, hospital equipment, and nutrition have an impact on the loyalty of patients (21). Other studies have identified parameters such as the presence of committed health care workers, access to adequate protective equipment, having a safe physical space, and meeting the basic needs of patients as the factors affecting patients' satisfaction (22). Access to protective equipment was identified as one of the most important needs in hospitals that provide care for COVID-19 patients. Therefore, ensuring access to an adequate supply of protective equipment is essential for the safety of health care staff as well as patients (23). Considering how hospitals needed to quickly set up new wards or change the application of their existing wards for treating COVID-19 patients, there were certain shortcomings and deficiencies in how the needs of critically ill patients were met, which indicates that hospitals were unprepared for such a crisis. 
Another requirement of the patients suffering from COVID-19 was education and the acquisition of credible information. Since COVID-19 is an emerging disease with unknown treatment, accurate and validated education is a critical component of all efforts to control this outbreak. However, in the age of massive social and information networks, not only is it difficult to distinguish between true and false information, but it is also imperative to avoid the negative psychological effects of overexposure to information (24). To tackle this problem, health care organizations, physicians, and nurses must be actively involved in these media and steer their audience toward credible sources (25). Medical personnel are also dutybound to get perfectly familiar with health precautions and standards and to teach them to patients and their families as well as ordinary people as much as possible, as people need and heed the guidance provided by healthcare professionals, particularly nurses. Several studies have shown the patients' need for specialized education about their diseases $(26,27)$. In the case of COVID-19, reliable information about the virus, how to protect ourselves against its exposure, and how to treat the infected is an essential educational need of these patients.

One of the most fatal complications of COVID-19 in patients with this disease is acute respiratory failure, which usually occurs approximately a week after the onset of symptoms and is characterized by rapid progression, followed by shortness of breath and hypoxemia. As a result, these patients need to receive specialized intensive care, including care under unstable conditions and continuous treatment by health care workers, and to be kept informed about their treatment process. Another need of these patients is access to committed specialized care of sufficient quality and quantity. Attempting various therapies, careful monitoring of the patient's condition, providing specialized nursing care, early detection of complications, and management of uncontrollable conditions have been reported to be the most important treatment measures for COVID-19 patients (28). All the above-mentioned results are consistent with the findings of this study.

In this study, the financial problems of COVID-19 for the patients, including the financial burden of treatment, the problems of returning to work, and the poor coverage of insurance companies regarding treatment costs and their need for social support, were placed in the support social needs category. Likewise, another study identified the nontransparent financial costs, disparities in the health care system, and work pressure on trained personnel as barriers to effective care and treatment, especially in patients in need of high levels of care. This study also showed that integrating social support with medical care and creating financial care guidelines will improve the quality of care (29).

The COVID-19 pandemic has created a global crisis of such severity that not only has necessitated drastic behavioral changes, but has also placed a heavy psychological burden on patients and their families. The cultural, social, and ethical implications and requirements of providing care and treatment to these patients have become more important with each passing day. Indeed, one of the im- portant needs of COVID-19 patients was found to be the need to have deep emotional interactions. Another study has also reported that compassionate empathy is among the most important types of interaction and relationship between the patient and health care workers in the present century (30). The success of health care workers in establishing a strong relationship with a sense of empathy with patients has been reported to have a great impact on patients' recovery, perceived self-value, distress, satisfaction, and hope (31). Additionally, it has been demonstrated that the patients who feel compassionate empathy from their physicians and medical staff are more successful in overcoming acute symptoms more quickly and have strengthened immune systems (32). COVID-19 patients must endure not only severe physical conditions, but also the stress of social and individual isolation due to the infectious nature of the virus, which disrupts their relationship with their family and friends and health care workers. Socially isolated patients are known to have a higher risk of perceived insecurity, physical drug side effects, fear of transmitting the disease to others, fear of negative news on social media, experience of loneliness, anxiety, stress, insomnia, and posttraumatic stress symptoms (33). Poor attention to the patients' needs to maintain deep emotional interactions can have wide-ranging psychological and emotional implications for these people (34).

Given the qualitative nature of the study, the small number of participants, and the selection of patients from the medical centers of 1 city, the findings may have limited generalizability to other locations. In this study, participants had a very unique experience due to COVID-19 disease and appropriate to Iranian culture. This was the first study that explained care needs and preferences based on COVID-19 patients' experiences. Considering the persistence of the COVID-19 pandemic, the results can be used to prepare care guidelines for achieving better outcomes for these patients. Thus, it is the most necessary step in improving nursing care quality at any critical situation. Future studies are recommended to design interventions based on these findings and examine their effect on the outcome of the disease.

\section{Conclusion}

COVID-19 patients experience a wide range of symptoms and problems which put them in need of extensive treatment and care. According to our findings, the most important care preferences for these patients are access to education and information from credible sources, access to specialized care, the fulfillment of support-social needs, and the fulfillment of the need for deep emotional interactions. Considering the ambiguous nature of COVID-19 and its extensive complications, the use of past experiences to meet these needs may be able to improve and enhance the results of treatment and care efforts for COVID19 patients.

\section{Acknowledgements}

We wish to acknowledge all the participants contributed in any way for the completion of this study. This project 
was completely supported and funded by Baqiyatallah University of Medical Sciences, Tehran, Iran. Thanks to guidance and advice from the "Clinical Research Development Unit of Baqiyatallah Hospital of Medical Sciences, Tehran, Iran.

\section{Ethics approval and consent to participate}

The present study was approved by the Ethics Committee of Baqiyatallah University of Medical Sciences, Tehran, Iran, with code IR.BMSU.REC.1399.026.

\section{Conflict of Interests}

The authors declare that they have no competing interests.

\section{References}

1. Lai CC, Shih TP, Ko WC, Tang HJ, Hsueh PR. Severe acute respiratory syndrome coronavirus 2 (SARS-CoV-2) and corona virus disease-2019 (COVID-19): the epidemic and the challenges. Int J Antimicrob Agents. 2020:105924.

2. Lee AM, Wong JG, McAlonan GM, Cheung V, Cheung C, Sham PC, et al. Stress and psychological distress among SARS survivors 1 year after the outbreak. Can J Psychiatry. 2007;52(4):233-40.

3. Moldofsky H, Patcai J. Chronic widespread musculoskeletal pain, fatigue, depression and disordered sleep in chronic post-SARS syndrome; a case-controlled study. BMC Neurol. 2011 Mar 24;11:37.

4. Al-Omari A, Rabaan AA, Salih S, Al-Tawfiq JA, Memish ZA. MERS coronavirus outbreak: Implications for emerging viral infections. Diagn Microbiol Infect Dis . 2019;93(3):265-85.

5. Ting YJT, Victor CCH, Rowena CPL. A survey of patient satisfaction with obstetric anaesthesia service in Tuen Mun Hospital, Hong Kong. Sri Lankan J Anaesthesiol. 2020; 28(1):36.

6. Bidmon S, Elshiewy O, Terlutter R, Boztug Y. What Patients Value in Physicians: Analyzing Drivers of Patient Satisfaction Using Physician-Rating Website Data J Med Internet Res. 2020;22(2):e13830.

7. Rantz MJ, Popejoy L, Vogelsmeier A, Galambos C, Alexander G, Flesner M, et al. Reducing avoidable hospitalizations and improving quality in nursing homes with APRNs and interdisciplinary support: lessons learned. J Nurs Care Qual . 2018;33(1):5-9.

8. Stewart K, Doody O, Bailey M, Moran S. Improving the quality of nursing documentation in a palliative care setting: a quality improvement initiative. Int J Palliat Nurs. 2017;23(12):577-85.

9. Livesay S, Zonsius M, McNett M. Evaluating data to guide care delivery: Quality improvement methods and implementation science. Data for Nurses: Elsevier; 2020. p. 59-86.

10. Hasanpoor E, Bahadori M, Yaghoubi M, Haghgoshayie E, Mahboub-Ahari A. Evidence-based management as a basis for evidence-based medical consultation. BMJ Evid Based Med. 2020;25(3):83-4.

11. Bahadori M, Yaghoubi M, Haghgoshyie E, Ghasemi M, Hasanpoor E. Patients' and physicians' perspectives and experiences on the quality of medical consultations: a qualitative study. Int $\mathrm{J}$ Evid Based Healthc. 2020;18(2):247-55.

12. Henry M, Alias A, Cherba M, Woronko C, Rosberger Z, Hier M, et al. Immediate post-treatment supportive care needs of patients newly diagnosed with head and neck cancer. Support Care Cancer. 2020:111.

13. Dahl TL, Vedsted P, Jensen H. The effect of standardised cancer pathways on Danish cancer patients' dissatisfaction with waiting time. Dan Med J. 2017;64(1):A5322.

14. Qiu HB, Li XY, Du B, Kang HYJ, Wang YS, Wang F, Sun B, Tong $\mathrm{ZH}$. [The keypoints in treatment of the critical coronavirus disease 2019 patient(1)]. Zhonghua Jie He He Hu Xi Za Zhi. 2020 Apr 12;43(4):273-277.

15. Sohrabi C, Alsafi Z, O'Neill N, Khan M, Kerwan A, Al-Jabir A, et al. World Health Organization declares global emergency: A review of the 2019 novel coronavirus (COVID-19). Int J Surg. 2020 Apr;76:7176.

16. Genzuk M. Qualitative research: An introduction to reading and appraising qualitative research. Occasional Paper Series Center for
Multilingual, Multiculture Research (Eds) Los Angeles, CA: Rossier School of Education, University of South California. 2009.

17. Delcourt C, Gremler DD, Van Riel AC, Van Birgelen M. Effects of perceived employee emotional competence on customer satisfaction and loyalty. J Serv Manage. 24(1):5-24.

18. Berry LL, Bendapudi N. Health care: a fertile field for service research. J Serv Res. 2007;10(2):111-22.

19. Maher A, Aghajani M, Ghotbi M, Barazandeh S, Safaei A, Anbari L. Managing and Improving the Quality of Hotel Services Through a Program to Improve the Quality of Hoteling in Government Hospitals in the Health Transformation Plan: Implementation Process, Results, and Challenges. Hakim Health Sys Res. 2017;20(2):99-109.

20. Braithwaite J, Mannion R, Matsuyama Y, Shekelle P, Whittaker S, Al-Adawi S. Health systems improvement across the globe: success stories from 60 countries: CRC Press; 2017.

21. Jafar Tajrishi M, Tabibi SJ. The effect of hoteling quality on patient loyalty in private hospitals of Tehran from patients' viewpoint. payavard. 2018;12(4):239-48

22. Sevin HD. Hotel Services In Hospitals. J Tourism Gastronomy Stud. 2018;6(1):451-9.

23. Huh S. How to train health personnel to protect themselves from SARS-CoV-2 (novel coronavirus) infection when caring for a patient or suspected case. J Educ Eval Health Prof. 2020;17.10.

24. Wen J, Aston J, Liu X, Ying T. Effects of misleading media coverage on public health crisis: A case of the 2019 novel coronavirus outbreak in China. Anatolia. 2020;31(2):331-6.

25. Merchant RM, Lurie N. Social media and emergency preparedness in response to novel coronavirus. JAMA. 2020.

26. Stirling BV, Harmston J, Alsobayel H. An educational programme for nursing college staff and students during a MERS-coronavirus outbreak in Saudi Arabia. BMC Nurs. 2015;14(1):20.

27. Moayed MS, Amoozadeh B, Parandeh A. Assessing health-care needs of patients with diabetes in Iran's health-care system: A modified Delphi method study. J Educ Health Promot. 2020;9.

28. Kleinpell RM. The role of the critical care nurse in the assessment and management of the patient with severe sepsis. Crit Care Nurs Clin North Am. 2003;15(1):27-34.

29. Long P, Abrams M, Milstein A, Anderson G, Apton K, Dahlberg M. Effective Care for High-Need Patients. Washington, DC. 2017.

30. Decety J. Empathy in Medicine: What It Is, and How Much We Really Need It. Am J Med. 2020;133(5):561-6.

31. Hua J, Howell JL, Sweeny K, Andrews SE. Outcomes of Physicians' Communication Goals During Patient Interactions. Health Commun. 2020:1-9.

32. Weilenmann S, Schnyder U, Parkinson B, Corda C, Von Kaenel R, Pfaltz MC. Emotion transfer, emotion regulation, and empathy-related processes in physician-patient interactions and their association with physician well-being: a theoretical model. Front Psychiatry. 2018;9:389.

33. Bo HX, Li W, Yang Y, Wang Y, Zhang Q, Cheung T, et al. Posttraumatic stress symptoms and attitude toward crisis mental health services among clinically stable patients with COVID-19 in China. Psychol Med. 2020:1-2.

34. Duan L, Zhu G. Psychological interventions for people affected by the COVID-19 epidemic. Lancet Psychiatry. 2020;7(4):300-2. 